\title{
EL IMPACTO de LAS NUEVAS TECNOLOGÍAs (TIC) EN LA CALIDAD DE LA CIUDADANÍA MEXICANA
}

\author{
Mtro. Francisco Abundis ${ }^{1}$
}

Resumen: En América Latina, la rápida expansión de las nuevas tecnologías (TIC) ha sido fundamental para diferentes sectores que buscan adaptarse a las condiciones sociales y económicas que la nueva sociedad de la información exige. En este proceso, los Estados han enfocado su modernización a la construcción de una nueva ciudadanía digital donde la relación con la sociedad, a través de medios electrónicos, sea más directa y transparente. Aunque los objetivos en educación no deben dejar de lado la alfabetización tecnológica de la población, es importante recordar que el principal reto que se enfrenta -en toda la región- sigue siendo la disminución de la brecha digital. Este trabajo comprobó que el uso de Internet juega un rol distintivo entre la población que puede tener o no acceso a ella. Se identificó un perfil sociodemográfico y político que muestra unos internautas jóvenes, mejor informados y más activos políticamente. Si bien es importante alimentar de herramientas a este grupo de usuarios, es necesario generar programas sociales y políticos que procuren nuevos caminos para la inclusión equitativa de todos los mexicanos a la sociedad de la información.

Abstract: In Latin America, the fast growing expansion of new technologies (ITC) is essential in various sectors that seek to adapt

\footnotetext{
Director Asociado, Parametría SA de CV. Candidato a Doctor en Ciencia Política por la Universidad de Connecticut, con énfasis en psicología social y método cuantitativo. Maestro en Políticas Públicas en Oxford. Maestro en Asuntos Internacionales en Columbia. Diplomado en Investigación Cuantitativa en Michigan. Diplomado en Sistema Político Estadounidense en California y Relaciones Internacionales en el Colegio de México. E-mail: fabundis@parametria.com.mx.
} 
to social and economic conditions that the new Information Society demands. In this process, States' modernization has focused in building a new digital citizenship where the relationship with society, through electronic media, becomes more direct and transparent. Although the educational goals should not ignore people's technological literacy, it is important to remember that the main challenge faced -in the entire region-remains to be the reduction of the digital divide. This study found that internet usage plays a distinctive role in the population that has or does not have internet access. This work identified a demographic and political profile that shows young internet users, as being better informed and more politically active. While it is important to continue providing tools for this user group, it is necessary to generate a social and political agenda that strives for new equitable and inclusive paths in order to integrate all Mexicans to the so called Information Society.

El proceso de globalización en América Latina ha promovido un acelerado desarrollo industrial y tecnológico en las tres últimas décadas. En este escenario, las tecnologías de la información y comunicación (TIC) han jugado un papel central como herramientas de facilitación de las relaciones entre los latinoamericanos y el mundo. El uso de estos nuevos instrumentos, aunado a la mayor productividad del capital y las nuevas reglas de flexibilización laboral, han impulsado transformaciones económicas, sociales y políticas en todo el globo, que afectan de manera diferencial a la población.

Sandoval y Mota (2007) explican que esta nueva sociedad de la información reúne diversos métodos de comunicación e información para ser manipulados a través de herramientas tecnológicas que se fusionan y convergen, con el fin de conformar una red de redes que le permite hoy al mundo facilitar diferentes procesos antes lejanos. De hecho, Castells (1999) afirma que esta sociedad hace uso de la informática y las tecnologías digitales como el motor de desarrollo de un nuevo esquema social que denomina como la "tercera ola". 
La importancia que adquieren hoy las TIC radica en su potencial como facilitadoras del desarrollo económico, social y político de las sociedades modernas. El abrir nuevos espacios de información y comunicación permite quebrar los limitantes geográficos que, en muchos casos, desventajan las posibles relaciones a nivel global y particular. Sin embargo, Sandoval y Mota (2007) advierten que si bien estos beneficios son necesarios dentro del proceso globalizador, el desarrollo y el aprovechamiento de este tipo de herramientas pueden generar consecuencias antagónicas que se traducirán en obstáculos en el progreso equitativo entre individuos y países.

Este posible desequilibrio social, consecuencia de la explotación desigual de los beneficios de las TIC, es hoy la mayor preocupación desde la ética democrática y progresista (Moreira, 2002). El aprovechamiento de estas nuevas tecnologías se da de manera diferenciada entre los sectores de la población que tienen acceso a ellas y quienes no. Un fenómeno global llamado "brecha digital" se convierte en la nueva forma de exclusión social que muestra "el rezago existente en un porcentaje considerable del mundo, en cuanto a acceso y aprovechamiento del Internet como medio de crecimiento económico y cultural". (Sandoval y Mota, 2007).

Los datos disponibles en las bases de datos de la CEPAL comprueban que el uso de estas tecnologías y el proceso de digitalización de los países han sido no sólo lentos, sino también desiguales. Entre el año 2002 y los datos más recientes del 2009, el porcentaje de latinoamericanos que tenían acceso a Internet se incremento apenas en 5 puntos porcentuales ( 8 y 13\%, respectivamente). ${ }^{2}$ En contraste, para el año 2002, en países como EE.UU. y Canadá,

Los resultados del 2009 pueden ser consultados en la página oficial de Estadísticas de la CEPAL, donde se reúne la información de encuestas 
poco más del $50 \%$ de la población tenía acceso a Internet (CEPAL, 2003); para el 2011, este porcentaje alcanza valores por encima del $75 \% .^{3}$ De hecho, las diferencias dentro de América Latina son notables: mientras que en países como Uruguay, Chile y Brasil los internautas son más de un tercio de la población, en países como El Salvador, Guatemala y Honduras este porcentaje es menos del 0,5\%. ${ }^{4}$

Asimismo, Tesoro (2005) señala que gran parte de esta brecha digital obedece a la alta concentración en la creación de nuevas tecnologías: el 66\% de las licencias y royalties tecnológicos en el mundo va a Estados Unidos y Japón, y el 79\% de los usuarios de Internet vive en países de la Organización para la Cooperación y el Desarrollo Económico (OCDE).

A pesar de las múltiples diferencias entre los países, la incursión de Internet como una de las herramientas más útiles en el proceso globalizador ha generado diversas expectativas en ciertos ámbitos. Así como las relaciones sociales entre individuos localizados en dos lugares opuestos en el mundo se han agilizado extraordinariamente, los intercambios económicos se han facilitado al punto de reducir de manera considerable sus costos de transacción. Estos impactos no son ajenos al ejercicio de la política y la democracia, donde la vinculación entre Estado y ciudadanos a través de medios electrónicos es el mecanismo más importante de la modernización del Estado.

de hogares de diferentes países en la región. Disponible en línea: http:// www.eclac.cl/tic/flash/

3 Información obtenida de la página oficial del Internet World Stats, para el 2011, con datos recopilados del US Census Bureau. Disponible en línea: http://www.internetworldstats.com/stats.htm

$4 \quad$ Estadísticas de la CEPAL (2011), op. cit. 


\section{Las TIC y la ciudadanía}

Son diversas las preguntas que surgen ante el uso de las TIC en la generación de impactos sociales y políticos en la formación de la ciudadanía. En esta construcción, los miembros de las sociedades deben jugar un rol activo dentro de la democracia. Ciudadanos mejor informados, con herramientas adecuadas para el aprovechamiento de oportunidades, preparados para la comunicación de ideas, dispuestos a velar por sus derechos y a controlar las acciones del Estado, son factores claves en el buen funcionamiento de la democracia. En la medida en que los individuos consigan el aprendizaje tecnológico necesario para el ejercicio de sus libertades, su participación política y la demanda de sus derechos, se podrán explotar las múltiples ventajas que las TIC facilitan en la relación entre ciudadano y Estado.

En esta línea, Sierra (2007) presenta a Internet como un nuevo espacio de interlocución y participación social que sugiere nuevas dinámicas de interacción entre los ciudadanos, y que exige al mismo tiempo un nuevo marco de desarrollo para las ciudades. En esta medida, una de las principales transformaciones asociadas al uso de las TIC es la nueva participación ciudadana, donde el espacio local se traslapa con un escenario global que abre nuevas vías de intercambio de información y comunicación entre los miembros de las sociedades. Este medio permitiría no sólo agilizar, sino también mejorar diferentes prácticas políticas ya en uso, tales como el voto o la representación política, así como generar otras nuevas que estrechen los vínculos entre ciudadanos y Estado.

Esta nueva posibilidad que abre Internet para formas más implicativas de participación política, explica Dahlberg (2001), es el resultado de la unión entre la estructura democrática de toda una comunidad política y el ejercicio de 
sus funciones de gobierno a través del uso de estas nuevas tecnologías. En esta misma línea, Subirats (2002) afirma que Internet no sólo juega el papel de mejorar las formas de participación democrática, sino que al mismo tiempo estrecha los lazos entre la ciudadanía y el gobierno. Coleman (2005) explica que este nuevo espacio en la democracia digital permite generar una representación permanente, mediante el continuo encuentro e intercambio comunicativo entre representante y representado. Acorde con Mansbridge (2003) y esta nueva dinámica en la participación democrática, el representante ajustará sus demandas con base en las opiniones y preferencias del potencial electorado, construyendo un nuevo sistema de representación donde el ciudadano podrá discutir los problemas públicos con el representante y con otros ciudadanos.

En esta medida, las palabras de López (2004) recuerdan la regla de oro de la garantía de este espacio común entre representante y representado:

Las administraciones locales tienen que garantizar (por ello) el acceso de los ciudadanos a la información gestionada por ellos, que los ciudadanos se informen, que los ciudadanos estén informados y que sean informados por iniciativas privadas y públicas. El derecho a la información tiene que hacerse realidad en el ámbito local porque es el derecho a la información local lo que hace que se haga eficaz la universalidad del derecho a la información.

En esta tarea, la Unión Europea lleva un largo camino recorrido. Desde el año 2001, la UE ha promovido el uso de las TIC en la administración pública con el fin de profundizar los esfuerzos de transparencia y participación pública emprendidos ya por diversos gobiernos. Finquelievich (2001) presenta algunos de estos adelantos en países de América Latina. Por un lado, en Buenos Aires se han desarrollado diferentes espacios virtuales que buscan una mayor participación ciudadana, sin embargo, 
esta vinculación no ha sido del todo exitosa, y el proceso de implementación tecnológica ha sido a paso lento. Asimismo, en Montevideo, los nuevos sistemas informáticos instalados no han resultado idóneos, debido a la falta de capacitación de los funcionarios que tienen que operarlos, lo cual limitó la articulación con la participación ciudadana a través de las TIC.

En contraste, Batista (2003) recoge diferentes experiencias de gobiernos latinoamericanos que señalan el uso de las TIC como herramientas viables y eficientes. Algunos de los casos donde la introducción de las TIC para mejorar el gobierno ha sido efectiva son países como Brasil, Perú, Ecuador y México, donde han concluido que la oportunidad y la conveniencia de implementar un programa de educación para el uso de las TIC juegan un papel esencial en el éxito de estos esfuerzos de promoción de la democracia digital.

La principal característica de la ciudadanía digital debe ser la apropiación de este espacio virtual con el fin de realizar sus actividades cívicas y sociales, más allá del simple uso de nuevas tecnologías. Empero, es importante contar con usuarios calificados que hagan uso constante de las herramientas TIC, y de usuarios comprometidos que conduzcan con éxito la representación permanente dentro del nuevo escenario de la democracia digital.

Es este último punto el que busca atender el presente artículo. Conocer el tipo de ciudadano que hace uso de Internet se convierte en la demanda más eminente en el plan de desarrollo de nuevos procesos de participación política digital e intercambio de información entre ciudadanos y Estado. Si bien diversas investigaciones han buscado exponer los múltiples beneficios derivados de la generación de nuevos espacios de comunicación entre la ciudadanía y el gobierno, son pocos los que presentan las características de los potenciales usuarios de tales herramientas. 
A través del levantamiento de una encuesta con representatividad nacional, se buscó indagar tanto sobre el uso de nuevas tecnologías tales como Internet, así como por aquellas diversas formas de participación política en las que se involucran los ciudadanos en México. Esta información nos permitió comprobar que los usuarios de Internet muestran no sólo un perfil sociodemográfico definido, sino también una formación política diferente a la población en general, y que fungirá como determinante esencial de las demandas futuras dentro de un posible espacio virtual de comunicación con sus representantes y el Estado. ${ }^{5}$

\section{Perfil sociodemográfico}

Robles y De Marco (2011) plantean que las demandas de los ciudadanos al Estado dependen, en gran medida, de las condiciones sociales y de la formación política de los usuarios de Internet. En esta medida, no es posible concebir al ciudadano digital como un ciudadano ordinario homólogo a la población general. Por el contrario, los ciudadanos que hacen uso de Internet cuentan con un perfil sociodemográfico bien definido. Casacuberta y Gutiérrez-Rubí (2010) presentan un estudio en España donde encuentran que es la población más joven (entre los 16 y los 24 años) la que representa el $70 \%$ de los usuarios de Internet en el país. Así como el $\mathbf{8 0 \%}$ de los blogueros resultan ser los menores de 35 años.

Parametría SA de CV es la casa encuestadora encargada del levantamiento. Son encuestas en vivienda con representatividad nacional. Las unidades de observación son seleccionadas a través de una muestra aleatoria simple de las secciones electorales reportadas por el IFE. Se realiza la encuesta a personas mayores de dieciocho años que residan en el lugar de interés, con un número total de mil casos. Levantamiento realizado: del 29 de octubre al 3 noviembre y del 3 al 8 de diciembre del 2011. 
Para México, los resultados de la encuesta nos presentan un primer panorama de los usuarios de TIC. Si bien el 50\% de los mexicanos afirma no haber utilizado nunca Internet, más del $20 \%$ afirma usarlo de forma constante y hasta el $29 \%$ de forma ocasional. A pesar de que la cifra de ciudadanos que aún no hace uso de esta herramienta tecnológica es muy alta, es importante rescatar el sector de población que parece estar involucrándose de forma ocasional con este instrumento. Sin embargo, al indagar sobre los principales temas de búsqueda cuando hacen uso de Internet, los resultados no parecen del todo alentadores. Tal como se ha mencionado anteriormente en América Latina, y se comprueba en este caso para México, el interés central de los internautas no es la política o la economía del país, sino más bien temas generales de salud, y en su gran mayoría, información relacionada con el entretenimiento.

Gráfico 1. ¿Usted ha utilizado alguna vez Internet? ¿Con qué frecuencia utiliza Internet en una semana? (porcentajes de población)

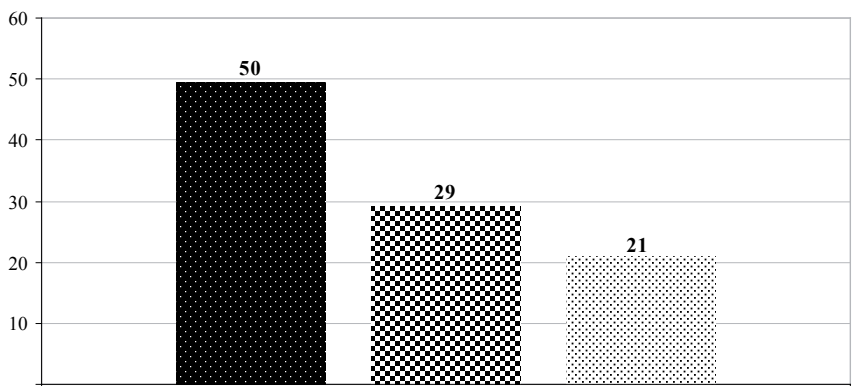

E No lo usa

Lo usa ocasionalmente

$\therefore$ Lo usa constantemente

Fuente: Encuesta en vivienda, Parametría SA de CV. Representatividad Nacional, octubre y noviembre de 2011. 
Gráfico 2. Cuando utiliza Internet, ¿con qué frecuencia realiza la búsqueda de la siguiente información? (porcentajes de población)

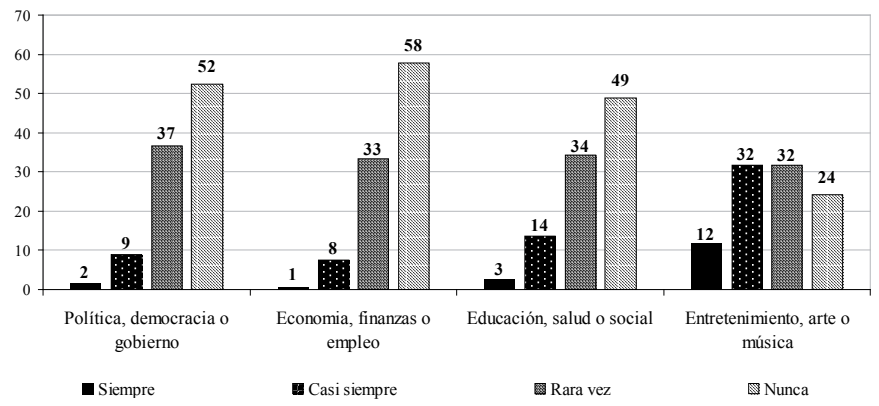

Fuente: Encuesta en vivienda, Parametría SA de CV. Representatividad Nacional, octubre y noviembre de 2011. Suman $100 \%$ con las respuestas No sabe / No contesta.

En lo que refiere a las características demográficas de los usuarios de Internet, los datos muestran que son los hombres, en su mayoría, quienes más utilizan esta herramienta tecnológica. Asimismo, el análisis por grupos etarios permite afirmar que son los mexicanos más jóvenes (entre los 18 y 25 años) quienes hacen mayor uso de Internet respecto a sus contrapartes de mayor edad. Acorde con los hallazgos en otros países, la juventud mexicana es la más involucrada con el uso de herramientas TIC en su vida diaria, y con ello, son también los más importantes posibles promotores de una ciudadanía digital futura. 


\section{Gráfico 3. Uso de Internet según género (porcentajes de población)}

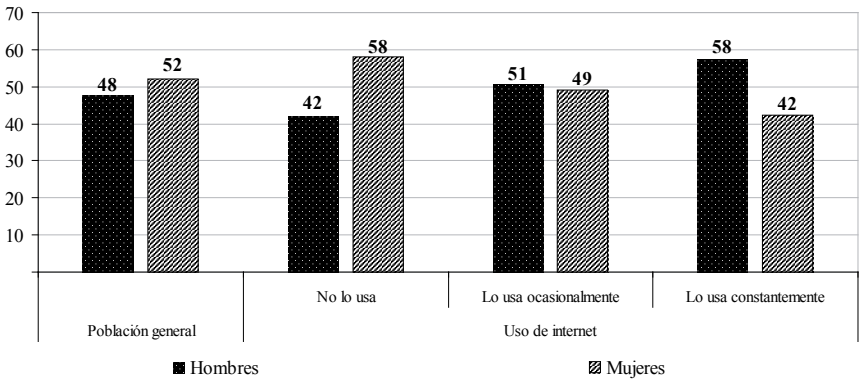

Fuente: Encuesta en vivienda, Parametría SA de CV. Representatividad Nacional, octubre y noviembre de 2011. Diferencia significativa al 95\% del N.C.

Gráfico 4. Uso de Internet según grupo etario (porcentajes de población)

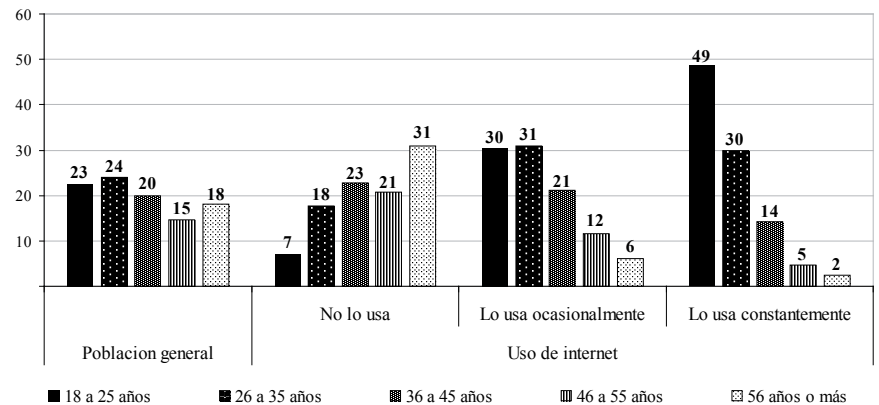

Fuente: Encuesta en vivienda, Parametría SA de CV. Representatividad Nacional, octubre y noviembre de 2011. Diferencia significativa al 95\% del N.C. 
Como se ha mencionado, el éxito en la implementación de la democracia digital requiere no sólo de internautas comprometidos con la construcción y el fortalecimiento de la ciudadanía digital, sino también de usuarios calificados para el uso de tales herramientas tecnológicas. En esta medida, no sorprende que los datos comprueben que a mayor escolaridad, se observe un uso de Internet mucho más extendido. Entre el 60 y el $80 \%$ de los mexicanos encuestados con preparatoria y universidad son quienes afirman utilizar de forma constante Internet. Asimismo, quienes viven en zonas urbanas y no se perciben a sí mismos como de clase baja son quienes responden que hacen uso de Internet ya sea de forma ocasional o cotidianamente. Cabe mencionar que lo anterior puede explicarse en que, en México aún hoy, Internet no es una herramienta disponible en muchas zonas rurales, o simplemente no está disponible para las personas de más bajos recursos.

Gráfico 5. Uso de Internet según nivel de escolaridad (porcentajes de población)

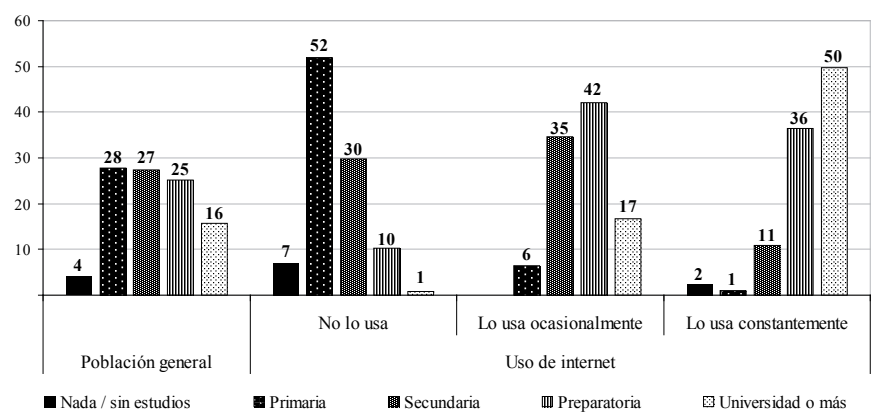

Fuente: Encuesta en vivienda, Parametría SA de CV. Representatividad Nacional, octubre y noviembre de 2011. Diferencia significativa al 95\% del N.C. 


\section{Gráfico 6. Uso de Internet según su percepción de clase $^{6}$ (porcentajes de población)}

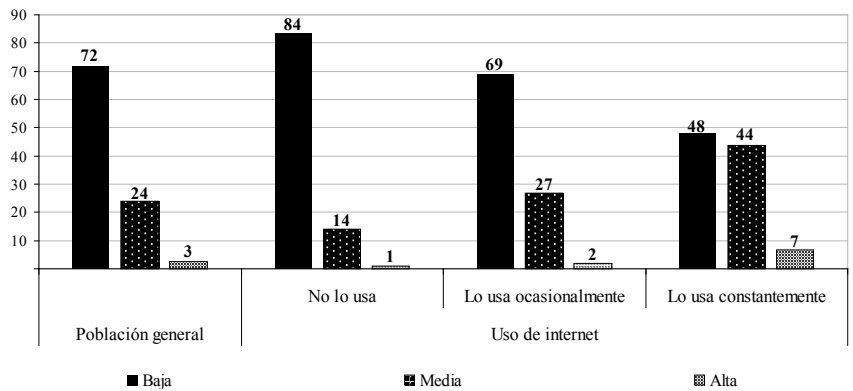

Fuente: Encuesta en vivienda, Parametría SA de CV. Representatividad Nacional, octubre y noviembre de 2011 . Suma $100 \%$ con las respuestas No sabe / No contesta. Diferencia significativa al 95\% del N.C.

\section{Gráfico 7. Uso de Internet según tipo de localidad (porcentajes de población)}

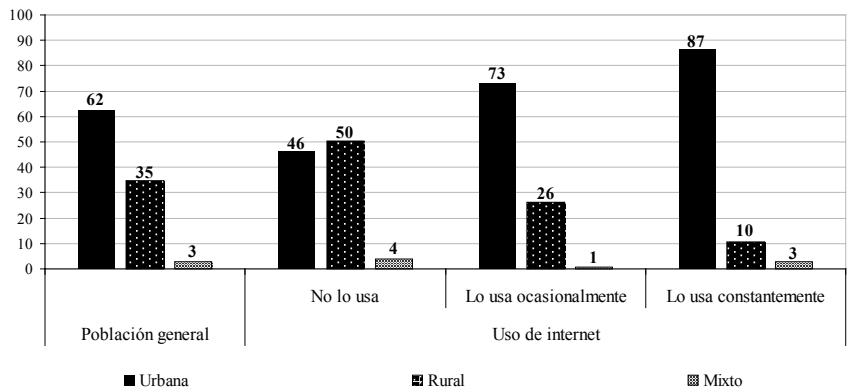

Fuente: Encuesta en vivienda, Parametría SA de CV. Representatividad Nacional, octubre y noviembre de 2011. Diferencia significativa al $95 \%$ del N.C.

$6 \quad$ Esta es una medida subjetiva que depende de la autopercepción de clase de los individuos encuestados. Se les pregunta: “ ¿Usted se considera de clase...?". Opciones de respuesta: baja, media-baja, media-media, media-alta, alta, NS/NC. 
En resumen, los datos de la encuesta levantada en México se equiparan a otros resultados encontrados en diversos países de América Latina. Debido a una mayor participación de los hombres en el mercado laboral, la información que manejan sobre las nuevas tecnologías parece extenderse de forma más general entre este grupo que entre sus contrapartes mujeres. Asimismo, son los mexicanos más jóvenes, con más altos niveles educativos, residentes de zonas urbanas y con una percepción de clase media o alta, quienes parecen estar más involucrados con el uso de Internet que otros grupos poblacionales.

\section{Perfil político}

Detrás de la irrupción de Internet como un nuevo espacio de participación política ciudadana y de posible fortalecimiento de los vínculos entre ciudadano y Estado, se crean diversas expectativas sobre los posibles usuarios atraídos por este nuevo escenario. Como se ha mencionado, el aprovechamiento de las herramientas tecnológicas buscaría incentivar una ciudadanía digital más activa en el funcionamiento de la democracia. Sin embargo, Lusoli, Ward y Gibson (2006) demuestran en su estudio entre la población inglesa que este tipo de redes sólo atrae a una pequeña minoría de votantes que, por lo general, son ya políticamente activos y privilegiados. A la vez, Di Gennaro y Dutton (2006) concluyen en su estudio que la participación política por Internet podría profundizar las ya existentes desigualdades sociales, marginando a quienes son más pobres y menos educados.

En otra línea, Casacuberta y Gutiérrez-Rubí (2010) muestran que en España, los usuarios de Internet son quienes se autoposicionan ideológicamente más a la izquierda que la población general, y quienes se interesan 
más por la política y lo social. Es decir, el uso de Internet significa para la mayoría un mayor activismo social (casi el 45\% de los usuarios afirma que participa en actividades de acción social).

Con el fin de conocer más de cerca el perfil político de los mexicanos que hacen uso de nuevas tecnologías, se presentan a continuación diversas respuestas que dan cuenta de las principales opiniones y actitudes políticas de este grupo. En primera instancia, fue posible comprobar que aquellos que hacen un uso más extendido de este medio de información tienden a evaluar más negativamente el rumbo del lugar donde viven (país y estado). Estas diferencias, sin embargo, son menores cuando se les pregunta por la especificidad de su estado que por el país. Esto puede explicarse en que la opinión de los internautas, tal como afirman Robles y De Marco (2011), está mediada por sus condiciones sociales, lo cual abre un espectro de respuestas muy amplio dadas las diferencias entre los estados en México.

Gráfico 8. Considerando todas las cosas, ¿cree usted que el país va por el camino correcto o por el camino equivocado? (porcentajes de población)

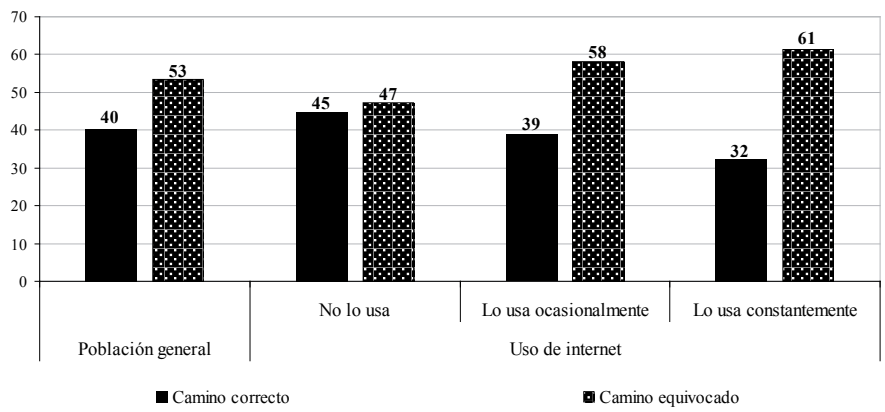

Fuente: Encuesta en vivienda, Parametría SA de CV. Representatividad Nacional, octubre y noviembre de 2011 . Suma $100 \%$ con 
las respuestas No sabe / No contesta. Diferencia significativa al 95\% del N.C.

\section{Gráfico 9. Considerando todas las cosas, ¿cree usted que su estado va por el camino correcto o por el camino equivocado? (porcentajes de población)}

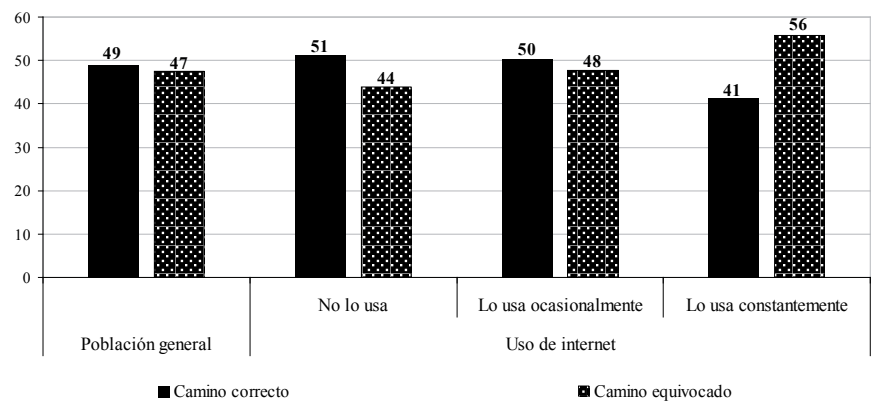

Fuente: Encuesta en vivienda, Parametría SA de CV. Representatividad Nacional, octubre y noviembre de 2011. Suma $100 \%$ con las respuestas No sabe / No contesta. Diferencia significativa al 95\% del N.C.

Asimismo, los resultados permiten observar que este pesimismo de los usuarios de Internet se extiende a su evaluación de la situación económica. Si bien tanto la población en general como quienes no usan Internet comparten la opinión negativa sobre el pasado y futuro de la economía del país, son quienes sí usan Internet los más pesimistas en esta evaluación. 
Gráfico 10. Durante los últimos doce meses, ¿usted diría que la economía del país ha mejorado o ha empeorado? (porcentajes de población)

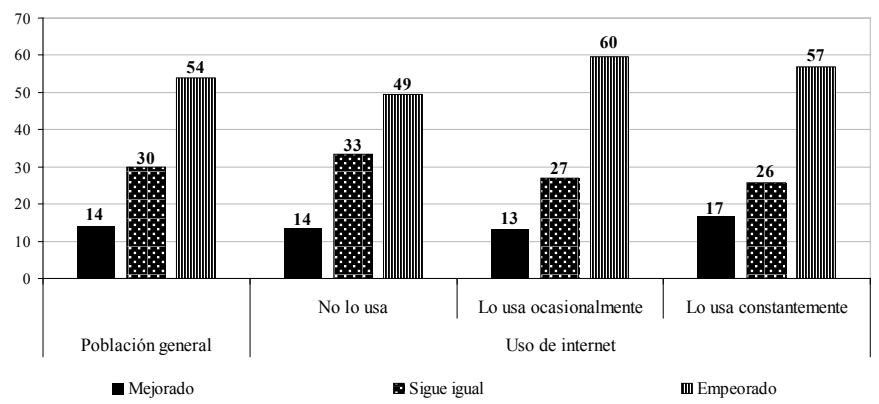

Fuente: Encuesta en vivienda, Parametría SA de CV. Representatividad Nacional, octubre y noviembre de 2011 . Suma $100 \%$ con las respuestas No sabe / No contesta. Diferencia significativa al 95\% del N.C.

\section{Gráfico 11. Pensando en los próximos doce meses, ¿usted diría que la economía del país mejorará o empeorará? (porcentajes de población)}

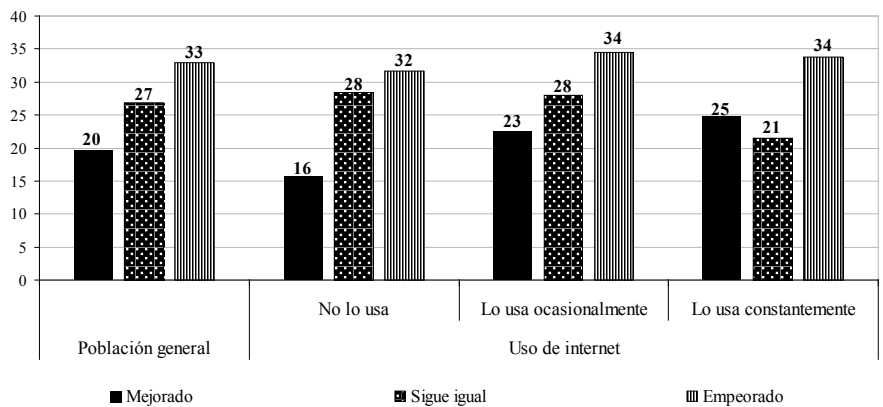

Fuente: Encuesta en vivienda, Parametría SA de CV. Representatividad Nacional, octubre y noviembre de 2011 . Suma $100 \%$ con las respuestas No sabe / No contesta. Diferencia significativa al 95\% del N.C. 
Aunque el presidente Calderón parece contar con la aprobación de más de dos tercios de la población mexicana, llama la atención que esta evaluación favorable disminuye en más de 10 puntos porcentuales entre quienes hacen mayor uso de nuevas tecnologías. Una realidad no muy distinta es la observada en la evaluación del trabajo del Gobernador del estado, quien se ve más desfavorecido por quienes usan más Internet.

Gráfico 12. En general, ¿usted aprueba o desaprueba la forma en que el presidente de la República Felipe Calderón realiza su trabajo? (porcentajes de población)

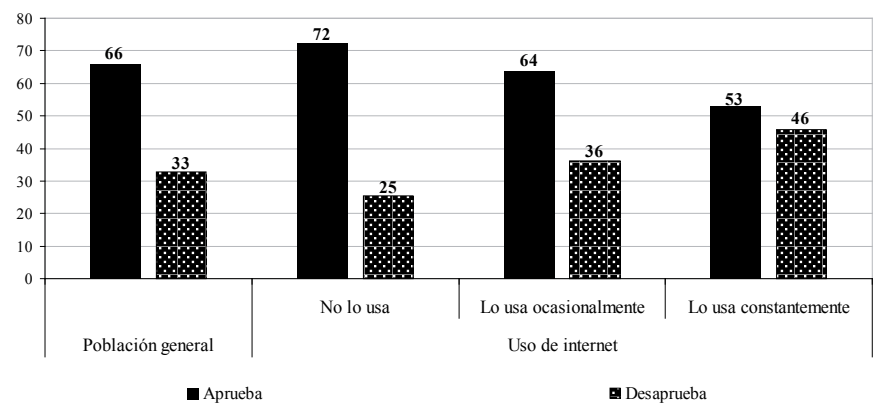

Fuente: Encuesta en vivienda, Parametría SA de CV. Representatividad Nacional, octubre y noviembre de 2011. Suma $100 \%$ con las respuestas No sabe / No contesta. Diferencia significativa al 95\% del N.C. 
Gráfico 13. En general, ¿usted aprueba o desaprueba la forma en que el Jefe de Gobierno / Gobernador de su estado realiza su trabajo? (porcentajes de población)

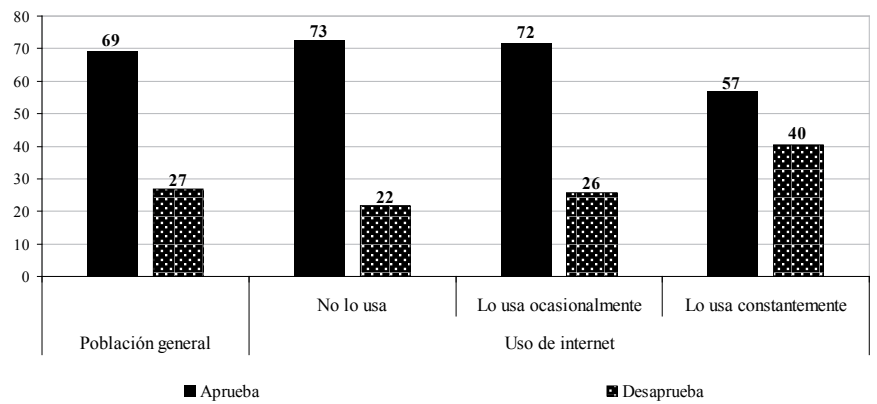

Fuente: Encuesta en vivienda, Parametría SA de CV. Representatividad Nacional, octubre y noviembre de 2011 . Suma $100 \%$ con las respuestas No sabe / No contesta. Diferencia significativa al $95 \%$ del N.C.

Posada (2011) afirma que los partidos y movimientos políticos son quienes se han apoderado de las herramientas de las TIC con el objetivo de reconectarse con el electorado, debido al déficit en la construcción de la participación democrática de los ciudadanos y, con ello, su paulatino distanciamiento de la política y de los asuntos públicos. El relativo alejamiento entre la política y los ciudadanos puede tener respuesta en los resultados obtenidos en la encuesta. En general, los mexicanos consideran que los problemas para entender los asuntos políticos se deben principalmente a la falta de interés de los ciudadanos y a la ausencia de mecanismos que, desde el Estado, busquen explicar tales temas a la sociedad. Esta primera explicación aumenta de manera significativa entre quienes usan de forma más regular Internet. 
Gráfico 14. Algunas de las personas dicen que tienen problemas para entender asuntos políticos y gubernamentales. En su opinión, ¿a qué se debe esto? (porcentajes de población)

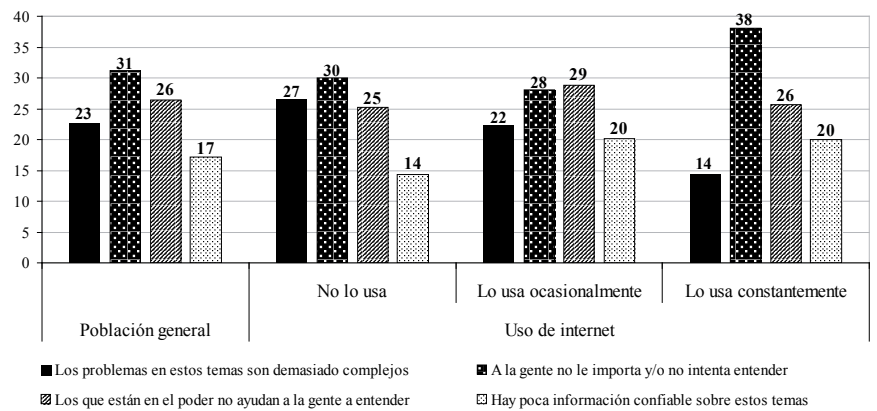

Fuente: Encuesta en vivienda, Parametría SA de CV. Representatividad Nacional, octubre y noviembre de 2011. Suma $100 \%$ con las respuestas No sabe / No contesta. Diferencia significativa al 95\% del N.C.

A pesar del aparente distanciamiento entre ciudadanos y la esfera política, los estudios de Scherman, Arriagada y Valenzuela (2010) entre jóvenes chilenos, y el de Bakker y De Vreese (2011) para el caso holandés, concluyen que los medios de información que consumen los jóvenes están fuertemente asociados a un mayor activismo político y social. Acorde con estos resultados, la encuesta revela que quienes usan con mayor constancia Internet suelen informarse de política a través de más de tres fuentes diferentes. Lo anterior revela que no sólo hacen un mayor uso de las potencialidades de las TIC, sino que a su vez buscan informarse sobre asuntos políticos por medio de fuentes alternativas, fortaleciendo con ello la ciudadanía digital que los convierte en protagonistas activos del funcionamiento democrático del país. Esta situación explica, a su vez, que a pesar de la percepción generalizada de que el gobierno no tiene en cuenta lo que opinan los ciudadanos, 
son quienes más usan Internet los que están menos de acuerdo con tal posición.

Gráfico 15. ¿Cuántas fuentes de información utiliza usted para informarse de política? (porcentajes de población)

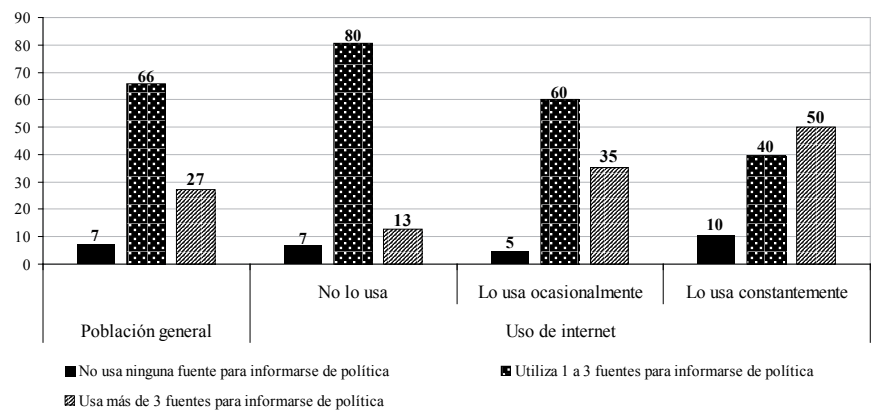

Fuente: Encuesta en vivienda, Parametría SA de CV. Representatividad Nacional, octubre y noviembre de 2011. Diferencia significativa al 95\% del N.C.

Gráfico 16. ¿Con cuál de las siguientes frases está usted más de acuerdo? (porcentajes de población)

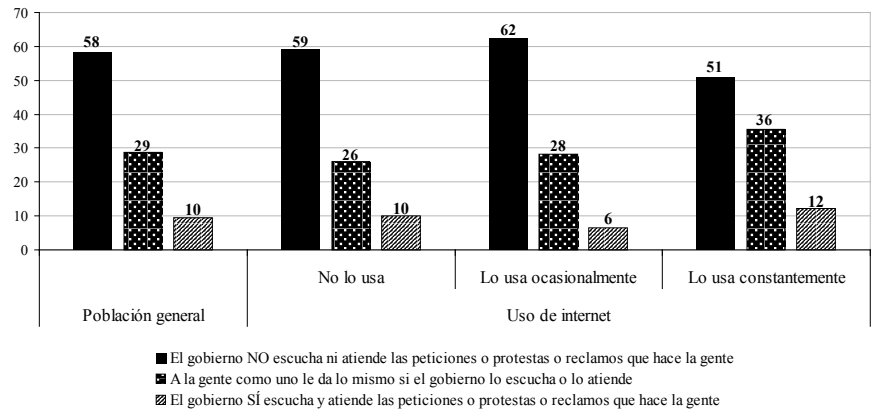

Fuente: Encuesta en vivienda, Parametría SA de CV. Representatividad Nacional, octubre y noviembre de 2011 . Suma $100 \%$ con las respuestas No sabe / No contesta. Diferencia significativa al 95\% del N.C. 
Si bien Tesoro (2005) afirma que Internet habilita una herramienta de empoderamiento de grupos y movimientos sociales a través del incremento de su capacidad operativa, la encuesta permite observar que, sin importar si hacen uso o no de la red, los mexicanos no se sienten seguros de influir, protestar y/o reclamar alguna decisión o ley del gobierno. Cabe destacar, empero, que quienes más usan Internet son quienes se perciben más seguros de poder hacer algo ante una situación de este tipo.

Gráfico 17. En el caso de que usted hiciera algo para influir, protestar y/o reclamar alguna decisión o ley del gobierno, ¿qué tan seguro está de tener éxito: seguro o inseguro? (porcentajes de población)

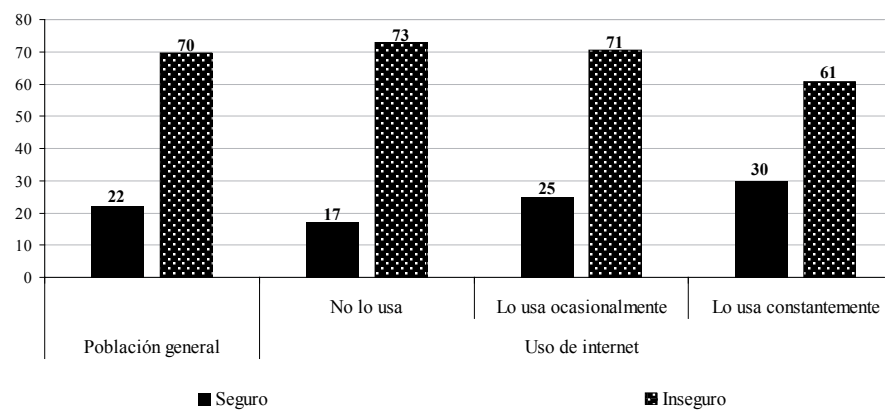

Fuente: Encuesta en vivienda, Parametría SA de CV. Representatividad Nacional, octubre y noviembre de 2011 . Suma $100 \%$ con las respuestas No sabe / No contesta. Diferencia significativa al 95\% del N.C.

En un escenario de baja participación política generalizado entre los mexicanos, se observa que quienes afirman utilizar con mayor concurrencia Internet participan, en mayor medida que otros grupos o que la misma población en general, en organizaciones de diferente índole. 
Cabe notar que la Iglesia se mantiene como la institución con mayor número de participantes, seguida tan sólo por organizaciones deportivas y recreativas. Este resultado pone en duda que una mayor alfabetización tecnológica derive en una ciudadanía más activa en lo político, ya que si bien la participación es mucho mayor entre quienes sí hacen uso de herramientas tecnológicas, ésta no parece tener como fin influir en la esfera política y con ello en el desarrollo de la democracia.

Gráfico 18. Participa / es miembro de alguna de las siguientes organizaciones (porcentajes de personas con respuestas afirmativas)

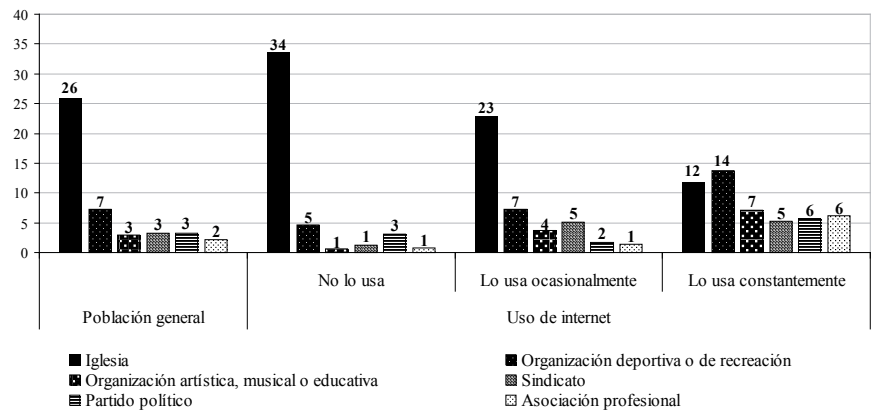

Fuente: Encuesta en vivienda, Parametría SA de CV. Representatividad Nacional, octubre y noviembre de 2011. Suma $100 \%$ para cada organización, con las respuestas negativas y las No sabe / No contesta. Diferencia significativa al $95 \%$ del N.C.

En cuanto a las acciones políticas directas para influir en las decisiones que toma el gobierno, las de mayor mención son ir a los medios de comunicación y reunir a personas interesadas formando un grupo. Si bien estas respuestas son generalizadas en la población, quienes hacen un mayor uso de Internet contemplan el trabajar a 
través de conexiones personales y familiares como uno de los métodos más efectivos para esta tarea.

Gráfico 19. Dígame por favor si considera usted efectivo cada uno de los siguientes métodos para influir en las decisiones que toma el gobierno (porcentajes de personas con respuestas afirmativas)

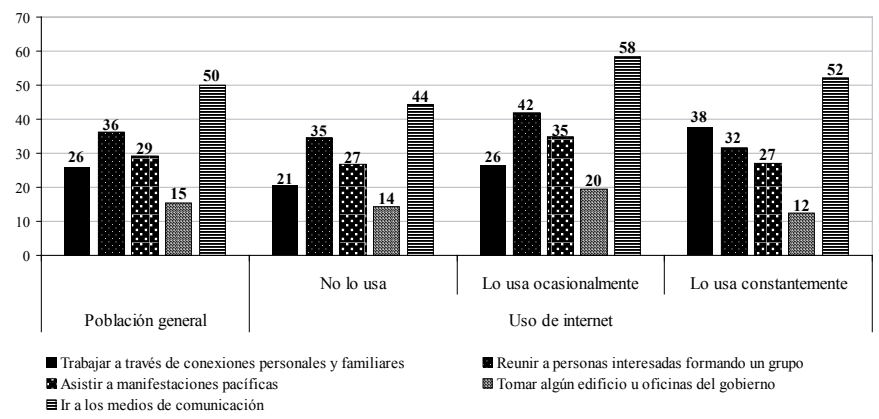

Fuente: Encuesta en vivienda, Parametría SA de CV. Representatividad Nacional, octubre y noviembre de 2011. Suma 100\% para cada acción, con las respuestas negativas y las No sabe / No contesta. Diferencia significativa al 95\% del N.C.

\section{Discusión y conclusiones}

Los resultados presentados hacen posible distinguir un perfil sociodemográfico y político definido de los mexicanos que hacen uso de nuevas tecnologías. En esta medida, los mexicanos más jóvenes y educados -que hacen uso de nuevas tecnologías- afirman informarse más de política a través de diferentes fuentes, a la vez que evalúan de forma más pesimista el trabajo de los funcionarios del gobierno y de la economía del país. Asimismo, las diversas variables presentadas en este trabajo muestran una relación 
significativa entre el perfil de quienes sí hacen uso de la red y los diferentes niveles de participación política organizativa o de acción directa con el fin de influir en materia de política.

Es importante mencionar que si bien el grupo de mexicanos que hace uso de Internet parece ser ya políticamente activo y crítico, es necesario promover iniciativas que busquen una mayor extensión del uso de las herramientas TIC, y con ello, favorecer la apertura de espacios para la participación ciudadana. ${ }^{7}$ En la medida que se restrinja el escenario virtual al principio de transparencia, se acotan los beneficios de una mayor participación política de los ciudadanos, y por consecuencia, de los vínculos de éstos con el Estado y sus representantes. En palabras de Negri y Hardt (2004): "Las nociones de rendición de cuentas y de gobernabilidad en esas propuestas van muy claramente encaminadas a asegurar la eficacia económica y la estabilidad, no a construir una forma representativa de control democrático".

En esta medida, el principal reto para la agenda pública de los municipios y de las instituciones políticas es redefinir el problema de la transparencia y la rendición de cuentas en un proceso abierto de comunicación directa y de acceso público entre los ciudadanos y el Estado. La generación de un nuevo espacio de intercambio de información entre representantes y representados sigue siendo una de las principales metas para los gobiernos de América Latina.

Como bien mencionan Casacuberta y GutiérrezRubí (2010), es necesario cambiar el paradigma en relación a las TIC y la participación política. Se trata de una

Porras, José Ignacio (2004), “Internet, política y democracia local”, Documentación Social 133, Chile, Universidad Bolivariana. Disponible en línea: http://www.esterkaufman.com.ar/sitios/kaufman/publicaciones/ ArtInternetyDemocraciaLocal.pdf 
transformación cultural y de hábitos en los que la forma de relacionarse se reestructura con el fin de ubicar al ciudadano digital como consumidor y productor de información relevante y necesaria para quienes se encuentran dentro del ejercicio político como representantes. Estos nuevos ciudadanos activos en la red deberán promover nuevas propuestas políticas de participación y representación, con el fin de generar mecanismos más inclusivos de nuevos grupos sociales antes marginados.

Si bien el proceso de modernización del Estado parece no haber alcanzado aún a los partidos políticos, éstos no pueden marginarse de tal proceso. La articulación de las estructuras partidarias como redes vivas de representación de un electorado juega un papel fundamental en el proceso de comunicación e intercambio de información con los nuevos ciudadanos digitales. En contraste, los movimientos sociales han aprendido a explotar los beneficios de las nuevas tecnologías en la generación de redes de comunicación, incluso de carácter global, que les permite hoy movilizar ciudadanos por distintas vías y en diferentes lugares del mundo.

¿Qué estamos proponiendo? Si bien el proceso de globalización tiende a marginar a quien no se adecua a los nuevos avances económicos, culturales, sociales y políticos, es necesario construir un plan de desarrollo integral incluyente dentro de la sociedad de la información. Ésta debe construir una ciudadanía digital que tenga cabida para todos los sectores sociales a través de programas de alfabetización tecnológica, en los que el acceso y la promoción en el mercado laboral sean equitativos para toda la población en conjunto. La participación política articulada de ciudadanos activos y calificados se convierte hoy en la primera necesidad del mercado de trabajo y en el proceso de modernización del Estado mexicano. 


\section{Bibliografía}

Bakker, T. y De Vreese, C. (2011), "Good News for the Future? Young People, Internet Use, and Political Participation", Communication Research, vol. 38, núm. 4. Disponible en línea: http://crx.sagepub.com/content/38/4/451. abstract.

Batista, Carlos (2003), "Las TIC para la gobernabilidad: la contribución de las tecnologías de la información y la comunicación a la gobernabilidad local en América Latina", Brasil, UNESCO, Universidad de Brasilia. Disponible en línea: http://portal.unesco.org/ci/en/ files/11316/10692492095Batista_report_esp_final.pdf/ Batista_report_esp_final.pdf.

Casacuberta, David y Gutiérrez-Rubí, Antoni (2010), "E-participación: de cómo las nuevas tecnologías están transformando la participación ciudadana”, Razón y Palabra, núm. 73, México, agosto-octubre. Disponible en línea: http://redalyc.uaemex.mx/src/ inicio/ArtPdfRed.jsp?iCve=199514908013.

Castells, Manuel (1999), La era de la información: economía, sociedad y cultura. Vol. 1 (La sociedad red), Madrid, Alianza Editorial, 590.

CEPAL (2003), Los caminos hacia una sociedad de la información en América Latina y el Caribe, CEPAL, Naciones Unidas, 136.

Coleman, Stephen (2005), “New Mediation and Direct Representation: Reconceptualizing Representation in the Digital Age", New Media \& Society, vol. 7, núm. 2.

Dahlberg, Lincoln (2001), "Democracy Via Cyberspace", New Media \& Society, vol. 3, núm. 2.

Di Gennaro, Corinna y Dutton, William (2006), “The Internet and the Public: Online and Offline Political Participation in the United Kingdom", Parliamentary Affairs, vol. 59, núm. 2. 
Estadísticas de la CEPAL (2011). Disponible en línea: http:// www.eclac.cl/tic/flash/

Finquelievich, Susana (2001), "Nuevas formas de gestión social: las tecnologías de información y comunicación (TIC) en los gobiernos locales de Buenos Aires y Montevideo", Seminario Regional de América Latina y el Caribe, Montevideo, Uruguay, UNESCO. Disponible en línea: http://www.documentacion.edex. es/docs/0201FINnue.pdf.

INTERNET WORLD STATS (2011), con datos recopilados del US Census Bureau. Disponible en línea: http:// www.internetworldstats.com/stats.htm.

López, Xosé (2004), Desafíos de la comunicación local. Guía para la práctica de la información en los ámbitos de proximidad, Sevilla, Comunicación Social Ediciones y Publicaciones.

Mansbridge, Jane (2003), "Rethinking Representation", American Political Science Review, vol. 97, núm. 4.

Moreira, Manuel (2002), "Igualdad de oportunidades y nuevas tecnologías: un modelo educativo para la alfabetización tecnológica", Revista de la Universidad Autónoma de Barcelona, Educar, núm. 29. Disponible en línea: http://ddd.uab.cat/pub/educar/0211819Xn29p55.pdf. Negri, Antonio y Hardt, Michael (2004), Multitud: guerra y democracia en la era del Imperio, Barcelona, Debate. Posada, Leydi (2011), “MIRA: Internet, participación y democracia. Las nuevas tecnologías y la reconexión con el ciudadano", Civilizar, vol. 11, núm. 20. Disponible en línea: http://www.usergioarboleda.edu.co/civilizar/ civilizar-20/MIRA.....pdf.

Robles, José Manuel y De Marco, Stefano (2011), "La participación digital y el comportamiento político de los usuarios de Internet: un acercamiento descriptivo a la ideología de los internautas", Papeles del CEIC, núm. 
65, Madrid, marzo. Disponible en línea: http://www. identidadcolectiva.es/pdf/65.pdf.

Sandoval, Eduardo y Mota, Laura (2007), "Indígenas y democracia en las tecnologías de información y comunicación (TIC)", México. Disponible en línea: http://www.ocyt.org.co/esocite/Ponencias_ ESOCITEPDF/1MEX059.pdf.

Scherman, Andrés; Arriagada, Arturo y Valenzuela, Sebastián (2010), “¿Hacia una nueva ciudadanía multifuncional? Uso de medios digitales, redes sociales online y participación política”, presentación para IV Congreso Latinoamericano de Opinión Pública de WAPOR, Brasil. Disponible en línea: http://uc-cl.academia. edu/Sebasti\%C3\%AlnValenzuela/Papers/905115/_ Hacia_una_nueva_ciudadania_multifuncional_Uso_ de_medios_digitales_redes_sociales_online_y_participacion_politica.

Sierra, Francisco (2007), "Nuevas tecnologías de la información y participación ciudadana: las políticas de gobernanza digital en Europa", Revista de Estudios para el Desarrollo Social de la Comunicación, Redes.com. Disponible en línea: http://www.revista-redes.com/ ojs/index.php/Redes-com/article/view/166.

Subirats, Joan (2002), "Los dilemas de una relación inevitable. Innovación democrática y tecnologías de la información y de la comunicación", Democracia Digital. Limites y oportunidades, Barcelona. Disponible en línea: http://www.comunitats.localret.cat/consensus/files/images/consensus/Innovaci\%C3\%B3n\%20 democr\%C3\%A1tica\%20y\%20TIC.pdf.

Tesoro, José Luis (2005), “Probidad, gobierno electrónico y modernización de la gestión pública en Iberoamérica: inferencias empíricas", Nueva Sociedad, núm. 195. Disponible en línea: http://www.nuso.org/upload/ articulos/3240_1.pdf. 